\title{
The transportation problem with exclusionary side constraints*
}

\author{
Dries Goossens ${ }^{\dagger}$ and Frits C.R. Spieksma ${ }^{\dagger}$
}

\begin{abstract}
We consider the so-called Transportation Problem with Exclusionary Side Constraints (TPESC), which is a generalization of the ordinary transportation problem. We determine the complexity status for each of two special cases of this problem, by proving NP-completeness, and by exhibiting a pseudo-polynomial time algorithm. For the general problem, we show that it cannot be approximated with a constant performance ratio in polynomial time (unless $\mathrm{P}=\mathrm{NP}$ ). These results settle the complexity status of the TPESC.
\end{abstract}

Keywords: Transportation problem; Exclusionary side constraints; Complexity

\section{Introduction}

The ordinary transportation problem is well-known: given a number of supply nodes each with a certain supply of items, a number of demand nodes each with a certain demand for items, and a unit transportation cost for each pair consisting of a supply node and a demand node, send the items from the supply nodes to the demand nodes at a minimum cost.

In this note we consider the variant where for each demand node a set of pairs of supply nodes is given such that at most one supply node of each given pair is allowed to send items to that demand node. Following the literature, we refer to this problem as the transportation problem with exclusionary side constraints (TPESC).

As far as we are aware, this problem has first been introduced by Cao [3] who described an application in storage management of containers. In this application, arriving containers must be positioned in rows of a storage yard, such that the costs of operations (searching, loading, retrieving) are minimized. Differences in size, ownership or content may disallow containers to be stored in the same row, giving rise to exclusionary side constraints. A branch-and-bound approach was described to solve the problem. Other branch-and-bound approaches are described and tested in Sun [10], while evolutionary algorithms have been proposed and tested by Cao and Uebe [4], and Syarif and Gen [11]. The complexity status of TPESC, however, has remained open.

${ }^{*}$ This research was partially supported by FWO Grant No. G.0114.03.

${ }^{\dagger}$ Department of Applied Economics, Katholieke Universiteit Leuven, Naamsestraat 69, B-3000 Leuven, Belgium. (e-mail: firstname.lastname@econ.kuleuven.be) 
Our interest in this generalization of the transportation problem stems from an application that occurs in the context of a procurement problem (see Section 2 and Goossens et al. [8] for a description).

\subsection{Problem Statement}

TPESC can be formulated as follows. Let there be a set $S$ of supply nodes, each with a supply of $s_{i}, i \in S$, and a set $D$ of demand nodes, each with a demand of $d_{j}, j \in D$. For each pair of consisting of supply node $i \in S$ and demand node $j \in D$, a unit cost $c_{i j} \geq 0$ is given. Finally, for each demand node $j \in D$, a (possibly empty) set of pairs of supply nodes, called $F_{j}$, is given; thus $F_{j}=\left\{\left(i_{1}, i_{2}\right) \mid\left(i_{1}, i_{2}\right) \in S \times S, i_{1} \neq i_{2}\right\}$. The problem is to send all supply to the demand nodes at minimum cost, such that each demand node $j \in D$ receives items from at most one supply node for each pair of supply nodes present in $F_{j}$. Obviously, if $F_{j}=\emptyset$ for all $j \in D$, the ordinary transportation problem arises. Notice that we assume that total supply equals total demand, that is $\sum_{i \in S} s_{i}=\sum_{j \in D} d_{j}$. For a mathematical formulation, we refer to Sun [10].

\subsection{Our results}

In this paper, we show that TPESC is NP-complete. In fact, the problem is NP-complete already for the smallest nontrivial case, that is, the case with two demand nodes. Even more specifically, we establish for each of two relevant special cases of TPESC its approximability status (we refer to Garey and Johnson [6] or Ausiello et al. [1] for an introduction to these issues). One special case concerns the setting where all $F_{j}, j \in D$ are equal (we refer to this case as TPESC with common exclusionary sets, see Section 2). Then, we show that in case of two demand nodes the problem is (weakly) NP-complete and a pseudo-polynomial time algorithm exists. In case of three demand nodes, the problem becomes strongly NPcomplete. Another special case concerns the setting where all sets $F_{j}, j \in D$ except one are empty (we refer to this case as TPESC with a single exclusionary set, see Section 3). Then, we show that the existence of a polynomial-time algorithm with a fixed performance ratio would imply $\mathrm{P}=\mathrm{NP}$, even in the case of two demand nodes. Our results motivate the use of heuristics and branch-and-bound approaches ([3], [10], [4], [11]) for solving large instances of the TPESC.

\section{TPESC with common exclusionary sets}

In this section we focus on the TPESC with common exclusionary sets. This special case arises in a procurement problem (see Goossens et al. [8]). We now proceed to describe this problem in more detail.

Consider a buyer procuring given amounts of different goods from different suppliers. Each of the suppliers uses a so-called total quantity discount policy to set the prices for the different 
goods; more in particular, each supplier distinguishes volume intervals on the total number of sold goods that determine the prices charged for each individual good. The resulting procurement problem (referred to as the TQD problem) is to obtain the given amounts of each of the different goods from the suppliers at minimum cost. Thus, a solution for an instance of this TQD problem prescribes how much items of each good are ordered from each supplier. In Chauhan et al. [5], a PTAS is described for a special case of the problem involving a single good. Observe that the TQD problem (as the TPESC) is a generalization of the ordinary transportation problem. Indeed, by associating a demand node to each good (with its demand equal to the amount that needs to be bought), and by associating a supply node with appropriate lower and upper bounds to each volume interval of each supplier, the TQD problem boils down to selecting supply nodes (at most one from each supplier) and by finding the right amount of goods to be transported. (In case a supplier can only deliver a fixed number of items, that is, there is only one supply node for each supplier with coinciding upper and lower bound, the ordinary transportation problem arises). One important aspect in this generalization of the transportation problem is the fact that for each demand node, a set of supply nodes is given (namely the nodes corresponding to the intervals of a single supplier) from which at most one can be used to actually supply that demand node; this corresponds to our $F_{j}, j \in D$ sets. Also, observe that these sets are the same for all demand nodes, in other words, we are dealing with an instance of TPESC with common $F$-sets.

Let us now formulate our results for this special case of TPESC. We first prove that the problem with $|D|=2$, that is, the case of two demand nodes, is weakly NP-complete, then we exhibit a pseudo-polynomial algorithm for this case, and finally we show that the problem with $|D|=3$ is strongly NP-complete.

Theorem 2.1 TPESC with common exclusionary sets is NP-complete, even if $|D|=2$.

Proof. We prove the theorem by presenting a reduction from Even-Odd Partitioning (EOP) to TPESC. EOP is proved to be NP-complete in Garey et al. [7].

\section{EOP}

Input: $n$ pairs of positive integers $I_{i}=\left(x_{2 i-1}, x_{2 i}\right), i=1, \ldots, n$.

Question: does there exist a partition of $\{1, \ldots, 2 n\}$ into disjoint subsets $A$ and $B$ with $\left|A \cap I_{i}\right|=\left|B \cap I_{i}\right|=1$ for $i=1, \ldots, n$, and with $\sum_{i \in A} x_{i}=\sum_{i \in B} x_{i}$ ?

For each integer in the input of EOP, we construct a supply node with supply equal to the value of the integer, that is, we set $S=\{1,2, \ldots, 2 n\}$ with $s_{i}=x_{i}$ for $i=1, \ldots, 2 n$. There are two demand nodes, each having demand $d_{1}=d_{2}=\frac{1}{2} \sum_{i=1}^{2 n} x_{i}$. We set $F_{1}=F_{2}=$ $\left\{\left(x_{2 i-1}, x_{2 i}\right) \mid i=1, \ldots, n\right\}$, implying that at most one supply node per pair is allowed to send items to that demand node. All costs are 0 . This completes the description of the instance of TPESC.

A yes-answer to the EOP instance directly corresponds to a feasible solution of the TPESC instance. Also, by observing the fact that the two demand nodes have common exclusionary constraints, it is clear that in any feasible solution of the TPESC instance, each supply node sends its entire supply to precisely one of the demand nodes which in turn corresponds to a yes-answer of the EOP instance. 
Of course, this result leaves the possibility open that a pseudo-polynomial time algorithm exists for TPESC with common exclusionary sets and two demand nodes. We will now describe such an algorithm.

We first construct a graph $G=(V, E)$. There is a node in $G$ for each supply node in the TPESC instance. Let there be an edge between each pair of nodes for which there is an exclusionary constraint in $F$. The resulting graph can be partitioned into a number of connected components $\left(V_{i}, E_{i}\right), i=1, \ldots, c$, such that there is no exclusionary constraint between two vertices in different sets $V_{i}$. Thus, each connected component is maximal. For each component $i$, there are only two possible ways of dividing the supply of the nodes of that component over the two demand nodes (as observed earlier, the fact that the two demand nodes have common exclusionary constraints and that total supply equals total demand, implies that in any feasible solution, each supply node sends its entire supply to precisely one of the demand nodes). Indeed, if we pick an arbitrary node of each component and call it the primal node of the component, we can either assign its supply to the first demand node or to the second. This choice determines to which demand nodes the supply of the other nodes of that component must be sent.

Suppose that assigning the supply of the primal node of component $i$ to the first demand node results in a total supply of $w_{i}$ being sent to the first demand node by the component $i$, and a remaining supply of $r_{i}=\sum_{j \in V_{i}} s_{j}-w_{i}$ being sent to the second demand node. Further, let $p_{i 1}\left(p_{i 2}\right)$ correspond to the total cost corresponding to component $i$, when the supply of the primal node is being sent to the first (second) demand node. We partition the set of components into two subsets as follows: $C_{1}=\left\{i \mid w_{i} \geq r_{i}\right\}$, and $C_{2}=\left\{i \mid w_{i}<r_{i}\right\}$. This allows us to define decision variables $x_{i}$ such that, for $i \in C_{1}: x_{i}=1(0)$ if the supply of the primal node is being sent to the first (second) demand node, and for $i \in C_{2}: x_{i}=1(0)$ if the supply of the primal node is being sent to the second (first) demand node. We can formulate the TPESC problem with common exclusionary sets and two demand nodes as follows:

$$
\begin{aligned}
\operatorname{minimize} & \sum_{i \in C_{1}}\left[p_{i 1} x_{i}+p_{i 2}\left(1-x_{i}\right)\right]+\sum_{i \in C_{2}}\left[p_{i 2} x_{i}+p_{i 1}\left(1-x_{i}\right)\right] \\
\text { subject to } & \sum_{i \in C_{1}}\left[w_{i} x_{i}+r_{i}\left(1-x_{i}\right)\right]+\sum_{i \in C_{2}}\left[r_{i} x_{i}+w_{i}\left(1-x_{i}\right)\right]=d_{1} \\
& \sum_{i \in C_{1}}\left[r_{i} x_{i}+w_{i}\left(1-x_{i}\right)\right]+\sum_{i \in C_{2}}\left[w_{i} x_{i}+r_{i}\left(1-x_{i}\right)\right]=d_{2} \\
& x_{i} \in\{0,1\} \text { for } i=1, \ldots, c .
\end{aligned}
$$

Let us now define for $i \in C_{1}: a_{i}=w_{i}-r_{i}, p_{i}=p_{i 1}-p_{i 2}$, and for $i \in C_{2}: a_{i}=r_{i}-w_{i}, p_{i}=$ $p_{i 2}-p_{i 1}$, and we define $B=d_{1}-\sum_{i \in C_{1}} r_{i}-\sum_{i \in C_{2}} w_{i}$.

Rewriting (1)-(4) using these definitions gives us the following equivalent integer program:

$$
\operatorname{minimize} \sum_{i=1}^{c} p_{i} x_{i}
$$




$$
\begin{array}{ll}
\text { subject to } & \sum_{i=1}^{c} a_{i} x_{i}=B \\
& x_{i} \in\{0,1\} \text { for } i=1, \ldots, c .
\end{array}
$$

Notice that when constraint (6) is satisfied, both the first and the second demand node receive their required supply of $d_{1}$ respectively $d_{2}$. Also observe that the definitions above imply that $a_{i} \geq 0$. In fact, we can eliminate those variables $x_{i}$ which have as coefficient $a_{i}=0$ (since, in an optimal solution we set, in case $a_{i}=0: x_{i}=1$ if $p_{i} \leq 0$, else we set $x_{i}=0$ ). Thus, henceforth we will assume that $a_{i} \geq 1$. Furthermore, we assume that $B \geq 0$, since no solution exists if $B<0$.

This problem is a generalization of the change making problem (see Martello and Toth [9]), since there is a cost $p_{i}$ associated to each variable $x_{i}$. Furthermore, there are bounds equal to 1 on the variables. Wright [12] developed a dynamic program for the change-making problem. The following modified version of this algorithm, to which we refer as algorithm DP, provides an optimal solution for formulation (5)-(7).

Let $f_{q}(z)$ be the optimal solution value of a sub-instance of (5)-(7), consisting of components $1, \ldots, q$ and a right-hand side of $z$, with $1 \leq q \leq c$ and $0 \leq z \leq B$. If no solution exists for a combination of values $q$ and $z$, then $f_{q}(z)=\infty$. It is clear that

$$
f_{1}(z) \begin{cases}=0 & \text { if } z=0 \\ =p_{1} & \text { if } z=a_{1} \\ =\infty & \text { if } z \neq a_{1}\end{cases}
$$

Now, $f_{q}(z)$ can be computed by considering increasing values of $q$ from 2 to $c$ and, for each $q$, increasing values of $z$ from 0 to $B$ as

$$
f_{q}(z) \begin{cases}=f_{q-1}(z) & \text { if } z=0,1, \ldots, a_{q}-1 \\ =\min \left(f_{q-1}(z), f_{q-1}\left(z-a_{q}\right)+p_{q}\right) & \text { if } z=a_{q}, \ldots, B\end{cases}
$$

The optimal solution value of formulation (5)-(7) is then given by $f_{c}(B)$. The time complexity of algorithm DP is $O(c B)$, which proofs that TPESC with two demand nodes with common exclusionary constraints can be solved in pseudo-polynomial time. We have shown the following:

Theorem 2.2 Algorithm DP is a pseudo-polynomial algorithm for TPESC with common exclusionary constraints and two demand nodes.

We now argue that it is unlikely that this algorithm can be extended to the case of three demand nodes by showing that TPESC with common exclusionary sets and three demand nodes is strongly NP-hard. 
Theorem 2.3 TPESC with common exclusionary sets is strongly NP-complete, even if $|D|=3$.

Proof. We prove the theorem by presenting a reduction from Graph 3-colorability ([6]) to TPESC.

\section{Graph 3-colorability}

Input: a graph $G=(V, E)$.

Question: is $G$ 3-colorable, that is, does there exist a coloring of the vertices of $G$ such that two connected vertices receive different colors, and such that no more than three different colors are used?

We build an instance of TPESC by having a supply node for every vertex of $V$, by having a supply node for every edge of $E$, and by having a single dummy node $d$. Thus $S=V \cup E \cup\{d\}$. Each supply node corresponding to a vertex or an edge of $G$ has $s_{j}=1, j \in S \backslash\{d\}$, the supply corresponding to the dummy node equals $s_{d}=2(|V|+|E|)$. There are three demand nodes, each having demand $d_{j}=|V|+|E|$. Let the two endpoints of an edge $e \in E$ be denoted by $v_{e}$ and $w_{e}$, and let $p_{e}$ be the supply node in $S$ corresponding to this edge $e$. For each edge $e$ in $G$ there are three pairs of supply nodes in $F$ :

$$
F=\left\{\left(v_{e}, w_{e}\right),\left(v_{e}, p_{e}\right),\left(w_{e}, p_{e}\right) \mid e \in E\right\}
$$

Further, all costs are 0. This completes the description of an instance of TPESC.

Suppose that $G$ admits a 3-coloring. We associate a different color to each of the three demand nodes. Next, we send the unit supply of each supply node corresponding to a vertex $v \in V$ to the appropriate demand node (the one with $v$ 's color in the coloring). The unit supply of a supply node corresponding to an edge $e \in E$ is sent to the demand node not receiving supply from supply nodes $v_{e}$ and $w_{e}$ (obviously, there is always one such node). We use the supply from the dummy node to satisfy all demand from the demand nodes exactly. Observe that we have satisfied the exclusionary constraints, and hence: we have a feasible solution to TPESC.

Suppose there is a feasible solution to TPESC. Consider a triple of supply nodes $\left(v_{e}, w_{e}, p_{e}\right)$ associated to edge $e$. Due to the choice for $F$, it follows that the supply of each of these three supply nodes is sent to a unique demand node. Thus, the supply of supply nodes that correspond to vertices in $G$ that are connected, goes to different demand nodes. Since there are three demand nodes, we have found a 3-coloring.

\section{TPESC with a single exclusionary set}

In this section we deal with a special case of TPESC, namely the problem that arises when exactly one $F$-set is nonempty. As we shall see, already this restricted version is hard to approximate, even for two demand nodes. First, we sketch an application of this special case of TPESC. 
When a company decides to store its goods, it basically has the choice between constructing its own private warehouse and renting a public warehouse. Assuming that there are seasonal changes in the need for storage space, Ballou [2] shows that it is advisable to make use of both options. This leaves the company with the problem of where to store what goods, minimizing the total cost. One can imagine that the public warehouse imposes constraints on what goods can be stored together (e.g. hazardous materials), whereas these constraints could be non-existing in a private warehouse, since this warehouse can be built specifically according to the (safety) needs of the company. This practical application boils down to a TPESC with only two demand nodes, where only one has a nonempty $F$-set (namely the demand node corresponding to the public warehouse).

Theorem 3.1 TPESC with a single exclusionary set does not admit a constant-factor approximation algorithm unless $P=N P$, even if $|D|=2$.

Proof. We prove the theorem by presenting a reduction from Independent Set (IS) to TPESC.

\section{Independent Set}

Input: a graph $G=(V, E)$ and an integer $K \leq|V|$.

Question: does there exist an independent set of cardinality at least $K$, that is, a subset $V^{\prime} \subseteq V$ with $\left|V^{\prime}\right| \geq K$, such that no two vertices in $V^{\prime}$ are joined by an edge in $E$ ?

For each vertex in $V$ we construct a supply node, with supply 1 ; thus $s_{j}=1$ for all $j \in V$. There are two demand nodes; the first one has demand $d_{1}=K$, the second has demand $d_{2}=|V|-K$. All costs equal 0 . The first demand node has a set of exclusionary constraints $F_{1}=\{(k, l) \mid k, l \in V \wedge(k, l) \in E\}$. The second demand node has no exclusionary constraints, that is, $F_{2}=\emptyset$.

We now show that the existence of a polynomial-time algorithm with a constant performance ratio for TPESC would imply $\mathrm{P}=\mathrm{NP}$.

Suppose that the instance of IS has a yes-answer, that is, there exists an independent set $V^{\prime}$ of cardinality at least $K$. In this case, given the construction of $F_{1}$, there exist $K$ supply nodes corresponding to nodes from the set $V^{\prime}$ that satisfy the exclusionary constraints. It is now easy to see that a solution where the supply of these nodes is sent to the first demand node and where the other nodes supply the second demand node, is a feasible solution to TPESC that has zero cost.

In case that the instance of TPESC has a yes-answer, one immediately observes that the supply nodes assigned to the first demand node corresponds to an independent set of size $K$.

Thus an algorithm with a constant performance ratio for TPESC would find a zero cost solution if one exists, and hence would be able to distinguish between the yes-instances and the no-instances of IS. 


\section{References}

[1] G. Ausiello, P. Crescenzi, G. Gambosi, V. Kann, A. Marchetti-Spaccamela, M. Protasi, Complexity and approximation. Combinatorial optimization problems and their approximability properties, Springer, 1999.

[2] R.H. Ballou, Business logistics management, Prentice Hall, 1998.

[3] B. Cao, Transportation problem with nonlinear side constraints a branch and bound approach, Zeitschrift für Operations Research 36 (1992) 185-197.

[4] B. Cao, G. Uebe, Solving transportation problems with nonlinear side constraints with tabu search, Computers and Operations Research 22 (1995) 593-603.

[5] S.S. Chauhan, A.V. Eremeev, A.A. Romanova, V.V. Servakh, G.J. Woeginger, Approximation of the supply scheduling problem, Operations Research Letters 33 (2005) 249-254.

[6] M.R. Garey, D.S. Johnson, Computers and intractability: A guide to the theory of NPcompleteness, W.H. Freeman, New York, 1979.

[7] M.R. Garey, R.E. Tarjan, G. Wilfong, One-processor scheduling with symmetric earliness and tardiness penalties, Mathematics of Operations Research 13 (1988) 330-349.

[8] D.R. Goossens, A.J.T. Maas, F.C.R. Spieksma, J.J. van de Klundert, Exact algorithms for a procurement problem with total quantity discounts, Research Report 0452, Department of Applied Economics, Katholieke Universiteit Leuven, 2004.

[9] S. Martello, P. Toth, Knapsack problems: algorithms and computer implementations, John Wiley and Sons Ltd, 1990.

[10] M. Sun, The transportation problem with exclusionary side constraints and two branchand-bound algorithms, European Journal of Operational Research 140 (2002) 629-647.

[11] A. Syarif, M. Gen, Solving exclusionary side constrained transportation problem by using a hybrid spanning tree-based genetic algorithm, Journal of Intelligent Manufacturing 14 (2003) 389-399.

[12] J.W. Wright, The change-making problem, Journal of the Association for Computing Machinery 22 (1975) 125-128. 